\title{
A Case Report of Hyperleukocytosis in An Extremely Low Birthweight Neonate
}

Mehwish S. Sheikh, MD

\begin{abstract}
:
Hyperleukocytosis is elevation of WBC $>100,000 / u L$. The condition can lead to significant morbidities and can be associated with neonatal sepsis, myeloproliferative disorders of Trisomy 21, Neonatal leukemia, or leukocyte adhesion defect. The diagnosis is challenging in extremely low birth weight premature neonate, and limited literature is found for the preterm population. We present a case of hyperleukocytosis in extremely preterm neonate managed conservatively with a good outcome.
\end{abstract}

\section{"Physiological leukocytosis, white blood} cell (WBC) up to 30,000/uL in the neonatal period is well reported. Leukemoid reaction is when WBC $>50,000 / \mathrm{uL}$ and not because of the myeloproliferative disorder. Hyperleukocytosis (WBC > 100,000/uL) is not as well reported in extremely premature neonates."

\section{Introduction:}

Physiological leukocytosis, white blood cell (WBC) up to 30,000/ $\mathrm{uL}$ in the neonatal period is well reported. Leukemoid reaction is when WBC $>50,000 / \mathrm{uL}$ and not because of the myeloproliferative disorder. Hyperleukocytosis (WBC > 100,000/uL) is not as well reported in extremely premature neonates. The condition may be associated with myeloproliferative disorder or $L A D$, requiring extensive workup and extended days of antibiotic therapy. Some literature supports the watchful waiting in the extremely premature neonate. We present a case of an extremely preterm neonate with hyperleukocytosis, managed conservatively.

\section{Case report}

An extremely preterm ( $\sim 25$ weeks gestation age) female born to a multigravida via vaginal delivery in breech presentation. Mother was admitted $>7$ days for PPROM (Preterm premature rupture of membrane). She had routine antenatal care and was doing well until her admission to the hospital with leakage of clear fluid. She received Mg SO4 per our protocol and antenatal steroids on admission (>7 days before delivery). Mother was Rh-negative, otherwise serology negative and GBS negative.

The NB was admitted with a birthweight of 700 grams. On admission, the baby had respiratory acidosis with a $\mathrm{pH}$ of 6.9 and a pCO2 of 108 . The baby was switched to HFJV at the time with improving gases.

Further labs revealed the WBC to be $87,000 /$ uL peripheral smear showed $2 \%$ bands, $4 \%$ promyelocyte, $1 \%$ myelocyte, $62 \%$ seg- mented neutrophil, $21 \%$ lymphocytes. Hb was $11.4 \mathrm{~g} / \mathrm{dl}$ and platelet $350,000 / \mathrm{uL}$.

The baby was started on antibiotics, Fluconazole, adequate hydration through IVF. Repeat CBC at 12 hours of life reported WBC to be $102,000 /$ uL. Peripheral smear showed $6 \%$ bands, $4 \%$ promyelocyte, $4 \%$ myelocyte, $68 \%$ segmented neutrophil, 4\% lymphocytes. Hb was $12.4 \mathrm{~g} / \mathrm{dl}$ and platelet $350,000 / \mathrm{uL}$. CRP $<0.1$. Arterial blood gas continued to improve. Clinically, the newborn did not show other signs of sepsis. Lactic acid was 1.84. LDH was $726 \mathrm{U} / \mathrm{L}$.

Pathology review reported Nucleated red blood cell (RBC) within range for age; the left shift was suggestive of a reactive, infectious, or therapeutic process.

The newborn was managed for possible complications of hyperleukocytosis with hyperhydration.

The baby received 36 hours of antibiotics with two doses of Fluconazole. Blood culture was negative.

Placental pathology was reported as placental vasculitis and fetal membranes with chorioamnionitis.

As this was deemed to be most likely a Leukemoid reaction, no further workup was done to investigate the etiology.

The baby was extubated on $~$ day of life (DOL) 21 and required DART therapy. The baby was Discharged home on RA with Mild bronchopulmonary dysplasia (BPD) at $\sim 40$ weeks of gestation. Stable grade 1 intracranial hemorrhage was present at discharge.

This is an interesting case of an EPT neonate presenting with hyperleukocytosis in the first three days of life without further significant signs of infection, managed conservatively, with a short duration of antibiotic therapy. The WBC trended down to normal range without further investigation within seven days.

\section{Discussion:}

Physiologic elevation of leukocytes $(<30,000 / \mathrm{uL})$ in the neonate is well known and is usually associated with a physiological surge of granulocyte colony-stimulating factor. It has been reported to be a predictor of survival in some premature newborns (1). Leukemoid reaction is defined as a WBC count of more than 50,000 / uL when associated with etiology other than malignancy. Hyperleukocytosis is defined as a WBC count of more than $100,000 / \mathrm{uL}$ and is seen in neonatal leukemia, Leukocyte adhesion defect, and Trisomy 21; management can be challenging in an EPT infant. A literature search is limited for hyperleukocytosis in (extremely low birth weight) ELBW population. We present a case of an

\section{Readers can also follow} NEONATOLOG via our Twitter Feed @NEO 
extremely preterm (EPT) ELBW infant with hyperleukocytosis at birth (early onset) with resolution within seven days while following a conservative management approach.

Antepartum factors such as steroid use, chorioamnionitis, funisitis, preterm rupture of the membrane are associated with hyperleukocytosis (2). Our patient was born with extreme prematurity. There was an administration of antenatal steroids $>7$ days before birth and evidence of histological chorioamnionitis.

Neonatal leukemia is extremely rare (3). Our patient did not have abnormalities of the blood cell lines (no thrombocytopenia or anemia), no significant findings on the exam such as hepatosplenomegaly, leukemia cutis suggestive of leukemia (3); hence we did not proceed with bone marrow biopsy. Close monitoring with daily a $\mathrm{CBC}$ was recommended per consultation with Hematology.

The transient myeloproliferative disorder has a $10 \%$ incidence in Trisomy 21 (4). It manifests in the neonatal period as hyperleukocytosis and hepatomegaly with increasing circulating blast cells (5). Our patient did not have physical features attributable to Trisomy 21 , which sometimes is difficult to assess in EPT. Also, only a few blast cells were seen on pathology review.

Leukocyte adhesion deficiency is described with an incidence of 1 in 1 million (6). LAD 1 is a severe form and can present as hyperleukocytosis, delayed cord separation, and poor wound healing. This defect is most often a diagnosis of infancy, and only two cases in the neonatal period are found in the literature (7).

The diagnosis for the above possible etiologies requires a considerable amount of blood draw, which is challenging in ELBW neonates. We chose to follow the newborn conservatively without further diagnostics.

\section{"We followed the American Committee of Fetus and Newborn 2014 recommendation (12). We treated the baby with 36-hour empiric therapy with extended-spectrum antibiotics. Blood culture was negative, as were inflammatory markers. There was no clinical progression."}

Infectious disease is the second leading cause of neonatal mortality worldwide, preceded only by complications related to preterm birth. Hyperleukocytosis can be a finding in neonatal sepsis. The overall incidence of neonatal sepsis ranges from one to five cases per 1000 live births (8). At the same time, antibiotic stewardship is encouraged to avoid unnecessary exposure to antibiotics in this fragile population. Prolonged use of broad-spectrum antimicrobials in NICU increases the risk of Candida colonization and invasive infection, necrotizing enterocolitis, late-onset neonatal sepsis, and death $(9,10,11)$. We followed the American Committee of Fetus and Newborn 2014 recommendation (12). We treated the baby with 36-hour empiric therapy with extended-spectrum antibiotics. Blood culture was negative, as were inflammatory markers. There was no clinical progression.

While following the trend of WBC, we continued to provide hyperhydration to our patient in the first three days of life of up to 120

Table 1

\begin{tabular}{|l|l|l|l|l|l|l|}
\hline & DOL 1 & DoL 2 & DOL 3 & DOL 4 & DOL 6 & DOL 9 \\
\hline WBC & 87.3 & 102.3 & 84.2 & 44.6 & 35.2 & 23.1 \\
\hline $\begin{array}{l}\text { Neutrophils, } \\
\text { segmented }\end{array}$ & 62 & 68 & 70 & 75 & 72 & 68 \\
\hline Bands & 2 & 6 & 5 & 2 & 1 & 1 \\
\hline Metamyelocytes & 0 & 2 & 4 & 0 & 0 & 0 \\
\hline Promyelocyte & 4 & 4 & 1 & 0 & 0 & 0 \\
\hline Myelocyte & 1 & 4 & 1 & 0 & 0 & 0 \\
\hline Lymphocyte & 21 & 4 & 8 & 14 & 12 & 21 \\
\hline $\begin{array}{l}\text { Reactive } \\
\text { lymphocyte }\end{array}$ & 0 & 0 & 0 & 0 & 4 & 3 \\
\hline Basophils & 0 & 0 & 0 & 0 & 0 & 0 \\
\hline Eosinophils & 0 & 0 & 0 & 1 & 1 & 0 \\
\hline Monocyte & 5 & 11 & 10 & 8 & 10 & 7 \\
\hline
\end{tabular}


$\mathrm{ml} / \mathrm{kg} /$ day while keeping close monitoring of fluid balance. Our patient did not require exchange transfusion, as can be performed for extreme cases (3).

Hyperleukocytosis is associated with significant short- and longterm morbidities such as IVH, pulmonary hemorrhage, respiratory failure, RDS, BPD, renal failure, and DIC $(13,14)$. Our patient did not have IVH at 7 and 28 days of life on screening US, no $\mathrm{AKI}$, was extubated at $21 \mathrm{DOL}$ after requiring DART therapy. The patient did require high-frequency ventilator support in the first seven days of life. These findings have a strong association with prematurity itself. It is hard to associate this even partially with hyperleukocytosis.

Our patient did require pharmacological therapy for clinically significant PDA. This situation may have been attributable to higher fluid intake in the first week of life; however, there is no clear association of clinically significant PDA for TFI of $<150 \mathrm{ml} / \mathrm{kg} /$ day in the first weeks of life.

\section{References:}

1. Rastogi S, Rastogi D, Sundaram R, Kulpa J, Parekh AJ. Leukemoid reaction in extremely low birth weight infants. Am J Perinatol. 1999;16:93-7. doi: 10.1055/s-2007-993843

2. Zanardo V. Vedovato S. Trevisanuto D.D. Suppiej A. Cosmi E. Fais GF Histological chorioamnionitis and neonatal leukemoid reaction in low-birth-weight infants. Hum Pathol. 2006; 37: 87-91. doi: 10.1016/j.humpath.2005.09.017.

3. Van der Linden $M H$, Creemers S, Pieters $R$. Diagnosis, and management of neonatal leukaemia. Semin Fetal Neonatal Med 2012;2013:192-5. DOI: 10.1016/j.siny.2012.03.003

4. Hitzler JK, Zipursky A. 2005. Origins of Leukemia in children with Down syndrome. Nat rev/cancer5:11-20. DOI: 10.1038/ nrc1525

5. Zipursky A, Brown E, Christensen H, Sutherland R, Doyle J. 1997. Leukemia and/or myeloproliferative syndrome in neonates with Down syndrome. Semn perinatology 21:97-101. DOI: 10.1016/s0146-0005(97)80025-0

6. Etzioni A, Doerschuk CM, Harlan JM. Of man and mouse: Leukocyte and endothelial adhesion molecule deficiencies. Blood. 1999;94:3281-8. ISSN 0006-4971, https://doi. org/10.1182/blood.V94.10.3281.422k37_3281_3288.

7. Alizadeh $P$, Rahbarimanesh AA, Bahram MG, Salmasian $H$. Leukocyte adhesion deficiency type 1 presenting as leukemoid reaction. Indian J Pediatr. 2007;74:1121-3

8. Weston EJ, Pondo T, Lewis MM, Martell-Cleary P, Morin C, Jewell B, Daily P, Apostol M, Petit S, Farley M, Lynfield R, Reingold A, Hansen NI, Stoll BJ, Shane AL, Zell E, Schrag SJ. The burden of invasive early-onset neonatal sepsis in the United States, 2005-2008. IPediatr Infect Dis J. 2011 Nov;30(11):937-41. doi: 10.1097/INF.0b013e318223bad2

9. Kuppala V.S., Meinzen-Derr J., Morrow A.L., Schibler K.R. Prolonged initial empirical antibiotic treatment is associated with adverse outcomes in premature infants. J. Pediatr. 2011;159(5):720-725. doi: 10.1016/j.jpeds.2011.05.033.

10. Saiman L., Ludington E., Dawson J.D., et al. Risk factors for Candida species colonization of neonatal intensive care unit patients. Pediatr Infect Dis J. 2001 Dec;20(12):1119-24. doi: 10.1097/00006454-200112000-00005. PMID: 11740316.

11. Cotten C.M., Taylor S., Stoll B., et al. Prolonged duration of initial empirical antibiotic treatment is associated with increased rates of necrotizing enterocolitis and death for extremely low birth weight infants. Pediatrics. 2009 Jan;123(1):58-66. doi: 10.1542/peds.2007-3423. PMID: 19117861; PMCID: PMC2760222.

12. Cotten C.M. Antibiotic stewardship: reassessment of guidelines for management of neonatal sepsis. Clin. Perinatol. 2015Mar;42(1):195-206, x. doi:0.1016/j.clp.2014.10.007. PMCID: PMC4888789.

13. R. Hsiao, S.A. Omar Outcome of extremely low birth weight infants with leukemoid reaction. Pediatrics. 2005 Jul;116(1):e43-51. doi: 10.1542/peds.2004-1379. Epub 2005 Jun 1. PMID: 15930185.

14. Ridvan Duran a, U" Ifet Vatansever O" zbek a, Nu" khet Aladag ${ }^{\circ}$, iftdemir a, Betu"l Acunas, a, Necdet Su"t. The relationship between leukemoid reaction and perinatal morbidity, mortality, and chorioamnionitis in low-birth-weight infants. International Journal of Infectious Disease, 14(11). Doi: 10.1016/j.ijid.2010.06.012

Disclosure: The author has no disclosures.

NT
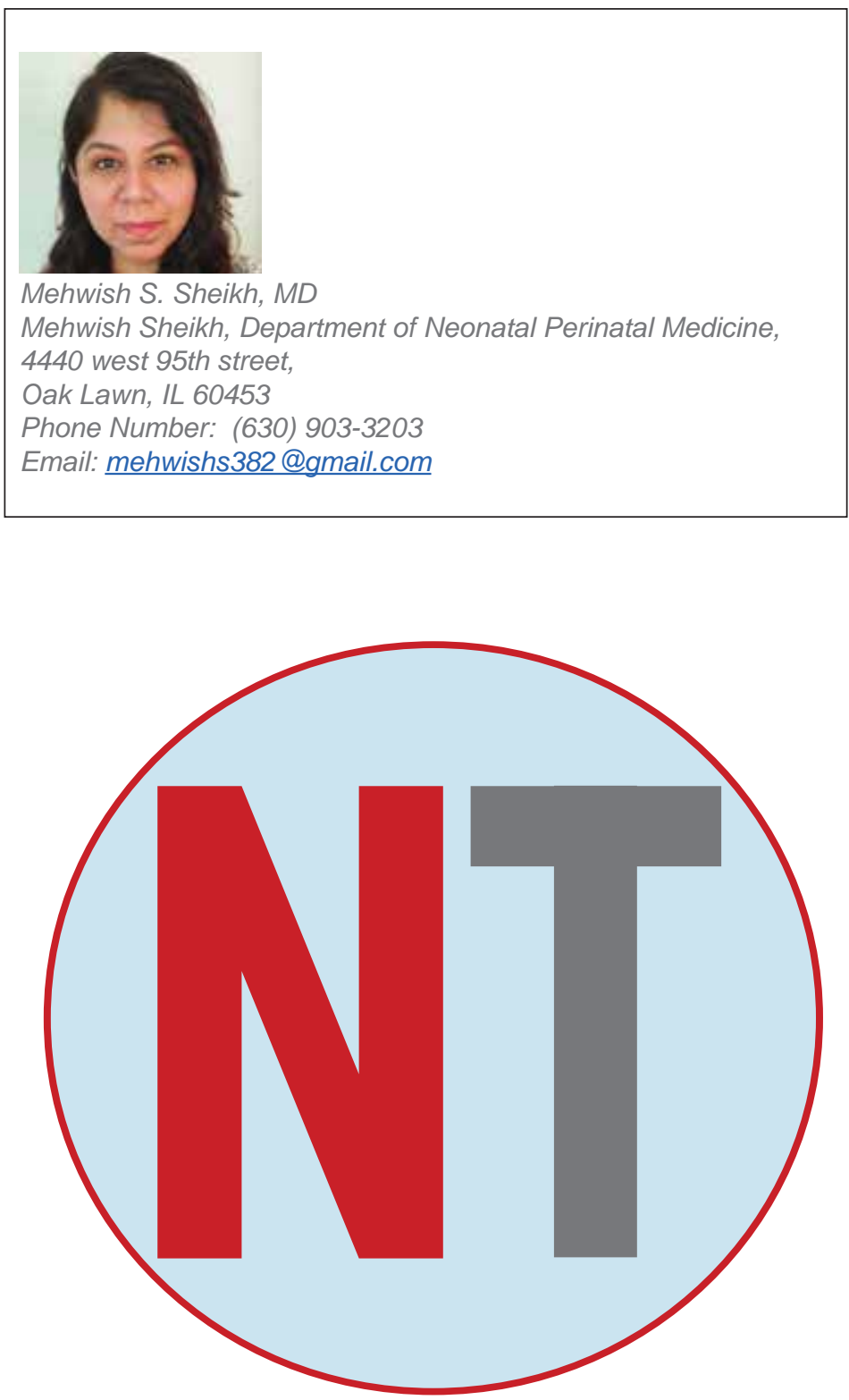